ESAIM: PROCEEDINGS, April 2012, Vol. 36, p. 217-228

Danièle Fournier-Prunaret, Laura Gardini, \& Ludwig Reich, Editors

\title{
ON GRADIENT-LIKE RANDOM DYNAMICAL SYSTEMS
}

\author{
Aya HMissi $^{1}$, FARIda Hmissi ${ }^{2}$ AND Mohamed HMissi ${ }^{3}$
}

\begin{abstract}
This paper deals with some characterizations of gradient-like continuous random dynamical systems (RDS). More precisely, we establish an equivalence with the existence of random continuous section or with the existence of continuous and strict Liapunov function. However and contrary to the deterministic case, parallelizable RDS appear as a particular case of gradient-like RDS.

The obtained results are generalizations of well-known analogous theorems in the framework of deterministic dynamical systems.
\end{abstract}

MSC (2000): Primary: 37H10. Secondary: 39B52, 37B25, 37B35

Keywords: Random dynamical system (RDS), Translation, Cocycle, Random homeomorphism, Gradientlike RDS , Random section, Liapunov function, Parallelizable RDS, Backward cocycle.

Résumé. Cet article est consacré à quelques caractérisations des systèmes dynamiques aléatoires (SDA) du type gradient. Plus précisément, nous montrons que cette notion est équivalente à l'existence d'une section aléatoire continue ou à l'existence d'une fonction de Liapunov stricte et continue. Mais, contrairement aux systèmes dynamiques déterministes, les SDA parallélisables apparaissent comme un cas particulier des SDA du type gradient.

Les résultats obtenus généralisent des théorèmes bien connus dans le cadre déterministe.

Mots clefs: Système dynamique aleatoire (SDA), Translation, Cocycle, Homomorphisme aleatoire, SDA du type gradient, Section aleatoire, Fonction de Liapunov, SDA parallélisable, Cocycle backward.

\section{INTRODUCTION}

Let $\phi$ be a continuous (deterministic) dynamical system on a locally compact space $E$, that is

(1) $\phi: \mathbb{R} \times E \rightarrow E,(t, x) \mapsto \phi(t, x)$ is continuous,

(2) $\phi$ satisfies the translation equation, i.e.

$$
\phi(0, x)=x, \quad \phi(s+t, x)=\phi(s, \phi(t, x)) ; \quad(s, t \in \mathbb{R}, x \in E) .
$$

Then $\phi$ is said to be gradient-like if there exists a set $S \subset E$ and an homeomorphism $h: E \rightarrow \mathbb{R} \times S$ such that $\phi(\mathbb{R}, S)=E$ and

$$
h(\phi(t, x))=(t, x) ; \quad(t \in \mathbb{R}, x \in S) .
$$

1 Département de Mathématiques, Faculté des Sciences de Tunis, Université de Tunis Elmanar, TN-2092 Elmanar, Tunis, Tunisia; e-mail: hmissi.aya@hotmail.com.

2 Département de Mathématiques, Faculté des Sciences de Tunis, Université de Tunis Elmanar, TN-2092 Elmanar, Tunis, Tunisia; e-mail: Farida.Hmissi@fst.rnu.tn.

3 Département de Mathématiques, Faculté des Sciences de Tunis, Université de Tunis Elmanar, TN-2092 Elmanar, Tunis, Tunisia; e-mail: Med.Hmissi@fst.rnu.tn.

(C) EDP Sciences, SMAI 2012 
By putting $Y:=\mathbb{R} \times S$ and $\kappa(t,(r, x)):=(t+r, x), h$ is in fact an homeomorphism between $\phi$ and the parallel dynamical system $\kappa$. In other words $\phi$ is parallelizable.

These notions were first introduced in [10] (cf. [2] and the related references). Following [2,5-7], the parallelization of $(E, \phi)$ is equivalent to each one of the following statements:

(1) $\phi$ possesses a continuous section, i.e. there exists a closed set $S \subset E$ and a continuous map $\tau: E \rightarrow \mathbb{R}$ such that $\phi(\tau(x), x) \in S$ for each $x \in E$.

(2) $\phi$ possesses a continuous strict Liapunov function, i.e. there exists a continuous map $l: E \rightarrow] 0, \infty[$ which is strictly decreasing along all trajectories of $\phi$.

(3) Each continuous cocycle $C: \mathbb{R} \times E \rightarrow] 0, \infty[$ of $\phi$ is of the form $C(t, x)=p(\phi(t, x)) / p(x)$ for some continuous $p: E \rightarrow] 0, \infty[$.

The aim of this paper, is to generalize this notion and those characterizations in the framework of random dynamical systems.

A continuous random dynamical system (RDS) with state space $E$, is defined as a pair $(\theta, \varphi)$ where $\theta$ is a translation on a measurable space $(\Omega, \mathcal{F})$ and $\varphi$ is a continuous cocycle over $\theta$. The paper is organized as follows: In the next section, we recall some useful notions related to random dynamical system. Then we adapt the deterministic case in order to introduce the notions of gradient-like random dynamical system, random section and random Liapunov function (although that the last notion has been already introduced in $[8,9]$ ).

The main result is then formulated in the last Section. Let $(\phi, \varphi)$ be a $\operatorname{RDS}$ with state space $E$. As in the deterministic case, we prove that the following assertions are equivalent:

(1) $(\theta, \varphi)$ is a gradient-like RDS.

(2) $(\theta, \varphi)$ possesses a continuous random section $S \subset \Omega \times E$.

(3) $(\theta, \varphi)$ possesses a continuous and strict Liapunov function

(4) The backward RDS associated to $(\theta, \varphi)$ is gradient-like.

However and contrary to the deterministic dynamical systems, parallelizable RDS appear as a particular case of gradient-like RDS. They correspond to gradient-like RDS for which the random section is a band (i.e. $S=\Omega \times Y$ for some $Y \subset E$ ). As in the deterministic case, we prove that a parallelizable RDS is random conjugate to a parallel RDS.

Finally, we apply our results to a model from random differential equations, namely the Lorenz system perturbed by real noises.

\section{Preliminaries}

In this paper, $(\Omega, \mathcal{F})$ is a measurable space, $\mathbb{R}$ denote the real line endowed with its $\sigma$-algebra $\mathcal{R}$ and $E$ is a locally compact space with countable base (LCCB) endowed with its Borel $\sigma$-algebra $\mathcal{E}$. The product $\Omega \times E$ is endowed with the usual $\sigma$-algebra $\mathcal{F} \otimes \mathcal{E}$. Moreover, $I_{Y}: Y \rightarrow Y$ denotes the identity map.

In the next section, we will give some preliminary definitions and remarks for later use. Firstly we give the definition of continuous random dynamical systems (cf [1] 1.1).

\subsection{Random Dynamical Systems}

A continuous random dynamical system (RDS) consists of two ingredients:

(1) A model of the noise, namely a measurable dynamical system (DS) $\theta$ defined on $(\Omega, \mathcal{F})$ : i.e. a mapping $\theta: \mathbb{R} \times \Omega \rightarrow \Omega,(t, \omega) \mapsto \theta_{t} \omega:=\theta(t, \omega)$ such that

(1) $\theta$ satisfies the translation equation, i.e.: For all $s, t \in \mathbb{R}, \omega \in \Omega$

$$
\theta_{0}=I_{\Omega} ; \quad \theta_{s+t}=\theta_{s} \circ \theta_{t} .
$$

(2) $(t, \omega) \rightarrow \theta(t, \omega)$ is $(\mathcal{R} \otimes \mathcal{F}, \mathcal{F})$-measurable. 
(2) A model of the system perturbed by noise, namely a cocycle on $E$ over the DS $\theta$, i.e.: A mapping $\varphi: \mathbb{R} \times \Omega \times E \rightarrow E,(t, \omega, x) \mapsto \varphi(t, \omega, x)$ such that

(1) $\varphi$ is $(\mathcal{R} \otimes \mathcal{F} \otimes \mathcal{E}, \mathcal{E})$-measurable.

(2) $(t, x) \rightarrow \varphi(t, \omega, x)$ is continuous for each $\omega \in \Omega$.

(3) The family $\varphi(t, \omega):=\varphi(t, \omega,):. E \rightarrow E$ of random mappings, satisfies the cocycle equation, i.e.: For $s, t \in \mathbb{R}, \omega \in \Omega$

$$
\varphi(0, \omega)=I_{E}, \quad \varphi(s+t, \omega)=\varphi\left(s, \theta_{t} \omega\right) \circ \varphi(t, \omega)
$$

In this case, we say that $\varphi$ is a continuous $\operatorname{RDS}$ driven by the $\operatorname{DS} \theta$, with state space $E$ and with parameters $\mathbb{R}$. It is denoted by $(\theta, \varphi)$.

Let $(\theta, \varphi)$ be a continuous RDS on $E$.We may associate the skew product $\Phi$ defined by

$$
\begin{array}{cccc}
\Phi: & \mathbb{R} \times \Omega \times E & \rightarrow & \Omega \times E \\
(t, \omega, x) & \rightarrow & \Phi_{t}(\omega, x):=\left(\theta_{t} \omega, \varphi(t, \omega, x)\right)
\end{array} .
$$

It can be easily verified that $\Phi$ is a measurable DS on the product space $\Omega \times E$.

\section{Remarks 1}

(1) Using the skew product, it can be easily verified that $t \mapsto\left(\theta_{t} \omega, \varphi(t, \omega, x)\right.$ is injective.

(2) It follows from (2) that $\varphi(t, \omega)$ is a homeomorphism of $E$ and

$$
\varphi(t, \omega)^{-1}=\varphi\left(-t, \phi_{t} \omega\right)
$$

(3) If $\Omega$ is reduced to one point (i.e. there is no noise), then the cocycle equation is reduced to the translation equation. Indeed, if $\Omega:=\{\varpi\}$ then $\theta_{t} \varpi=\varpi$ for $t \in \mathbb{R}$. Therefore, by putting $\varphi_{t}:=\varphi(t, \varpi)$, the relation (2) becomes

$$
\varphi_{0}=I_{E}, \quad \varphi_{s+t}=\varphi_{s} \circ \varphi_{t}
$$

which is the translation equation on $E$.

Hence, RDS generalize in a natural way the deterministic DS.

(4) The skew product allows natural generalizations from the deterministic case to the random case.

(5) Most of the time, RDS are defined over probability spaces. This is essentially connected to the concept of behaviors of RDS at infinity. Since we don't have such problems here, we omit the probability throughout this paper.

Examples 1 Let $\theta$ be a DS over a measurable space $(\Omega, \mathcal{F})$.

(1) Let $\pi$ be a continuous (deterministic) dynamical system on a LCB $E$. Obviously, $(\theta, \pi)$ is a continuous RDS with state space $E$.

Notice that, in this case, the associated skew product is in fact a diagonal product. It is given by

$$
\Pi(t, \omega, x)=\left(\theta_{t} \omega, \pi_{t} x\right) ; \quad(t \in \mathbb{R}, x \in E, \omega \in \Omega) .
$$

This means that the noise $\theta$ is not perturbed by the system $\phi$.

(2) If $E=\mathbb{R} \times Y$ for some LCCB space $Y$ and if $\pi: \mathbb{R} \times \Omega \times \mathbb{R} \times Y \rightarrow \mathbb{R} \times Y$ is given by

$$
\pi(t, \omega, r, y):=(t+r, y) ; \quad(t, r \in \mathbb{R}, y \in Y, \omega \in \Omega)
$$

then $(\theta, \pi)$ is called a parallel RDS generated by $Y$. 
Example 2 RDS (with continuous time) are models of solutions of random differential equations and stochastic differential equations. Next, we will only investigate the first case. For the second case, we need to introduce some non-trivial concepts (Wiener shift, stochastic integral ...) and this is not the aim of our paper. However, for the two cases, we refer the reader to the monograph [1].

Let $\theta$ be a DS over a measurable space $(\Omega, \mathcal{F})$, let $E$ be an open set of $\mathbb{R}^{d}$ and let $f: \Omega \times E \rightarrow E$ be measurable and such that for each $\omega \in \Omega$ :

(1) $(t, x) \rightarrow f\left(\theta_{t} \omega, x\right)$ is continuous.

(2) $x \rightarrow f\left(\theta_{t} \omega, x\right)$ is locally-lipschitz for each $t \in \mathbb{R}$.

Then the random differential equation

$$
\frac{d}{d t} X_{t}(\omega)=f\left(\theta_{t} \omega, X_{t}\right) ; \quad X_{0}(\omega)=x \in E
$$

admits a unique solution $\varphi$ which is a continuous RDS. The solution is given implicitly by

$$
\varphi_{t}(\omega) x=x+\int_{0}^{t} f\left(\theta_{s} \omega, \varphi_{s}(\omega) x\right) d s .
$$

\subsection{RDS-isomorphism}

Let $\varphi$ and $\psi$ be two RDS over the same driving DS $\theta$, but with state spaces $E$ and $F$ respectively. The RDS $\varphi$ and $\psi$ are said to be topologically equivalent or $R D S$-isomorph or random conjugate if there exists a mapping $f: \Omega \times E \rightarrow F$ such that

(a) For each $\omega \in \Omega$, the mapping $x \mapsto f_{\omega}(x):=f(\omega, x)$ is an isomorphism from $E$ onto $F$.

(b) For all $x \in E, y \in E$, both mappings $\omega \mapsto f(\omega, x)$ and $\omega \mapsto f_{\omega}^{-1}(y)$ are measurable.

(c) The cocycles $\varphi$ and $\psi$ are cohomologous; i.e., for all $\omega \in \Omega, x \in E$ and $t \in \mathbb{R}$ :

$$
\psi(t, \omega) f(\omega, x)=f\left(\theta_{t} \omega, \varphi(t, \omega) x\right) .
$$

In this case, $f$ is called a random isomorphism or a $R D S$-isomorphism.

\section{Remarks 2}

(1) This notion is classical even for RDS (cf. [1] 1.9.2). However, for the preceding convenient definition, we refer the reader to [3].

(2) Although it is not obvious but the random conjugaison is in fact an equivalence relation on the set of continuous cocycles driven by the same noise.

(3) If there is no noise (i.e $\Omega:=\{\varpi\}$ ), then $\varphi$ and $\psi$ will be deterministic DS on $E$ and $F$ respectively (cf. Remark 1). Let $\varphi_{t}:=\varphi(t, \varpi), \psi_{t}:=\psi(t, \varpi)$ and $u:=f(., \varpi): E \rightarrow F$, then (5) becomes

$$
\psi_{t}(u(x))=u\left(\varphi_{t}(x)\right) ; \quad(t \in \mathbb{R}, x \in E) .
$$

However, (6) means that the deterministic DS $\varphi$ and $\psi$ are topologically equivalent (or conjugate) in the classical sense (cf. [4] for example).

Hence, random isomorphisms (for RDS) generalize the classical notion of SD-isomorphisms.

\section{Characterizations of Gradient-Like RDS}

Let $(\Omega, \mathcal{F})$ be a measurable space and let $(E, \mathcal{E})$ be a LCCB space. We denote by $d$ a metric on $E$ which generates $\mathcal{E}$ and by $\mathcal{P}(E)$ the set of all subsets of $E$.

If $A \subset \Omega \times E$, let $A(\omega):=\{x \in E:(\omega, x) \in A\}$ the $\omega$-section of $A$. 
Note that, the set $A$ is identified with the set-valued map also denoted by $A: \Omega \rightarrow \mathcal{P}(E), \omega \mapsto A_{\omega}:=A(\omega)$.

A measurable set $A \in \mathcal{F} \otimes \mathcal{E}$ is called a random set if $\omega \rightarrow d\left(x, A_{\omega}\right)$ is measurable for any $x \in E$. A random set $A$ is said to be closed if $A_{\omega}$ is closed for every $\omega \in \Omega$.

For example, for each measurable subset $Z$ of $E$, the band $A:=\Omega \times Z$ is a measurable random set, closed whenever $Z$ is closed in $E$. In this case, $A(\omega)=Z$ for each $\omega \in \Omega$.

\subsection{Random section}

Let $(\theta, \varphi)$ be a continuous RDS on $E$.

\section{Definition 1}

(1) A random set $S \in \mathcal{F} \otimes \mathcal{E}$ is called a random section of the $\operatorname{RDS}(\theta, \varphi)$ if, for each $(\omega, x) \in \Omega \times E$ there exists a unique $\tau(\omega, x) \in \mathbb{R}$ such that

$$
(\theta(\tau(\omega, x), \omega), \varphi(\tau(\omega, x), \omega) x) \in S
$$

In this case, the section is also denoted by $[S, \tau]$.

(2) A random section $[S, \tau]$ of $(\theta, \varphi)$ is said to be continuous if, for every $\omega \in \Omega$, the function $x \rightarrow \tau(\omega, x)$ is continuous.

The proof of the following result is adapted from that of the deterministic case (cf. [2], Lemma 2.3).

Lemma 1 If $[S, \tau]$ is a continuous random section of $(\theta, \varphi)$, then the random set $S$ is closed.

Proof. Let $\omega \in \Omega$ and let $\left(x_{n}\right) \subset S(\omega)$ such that $\left(x_{n}\right) \rightarrow x \in E$. Then Since $\tau\left(\omega, x_{n}\right)=0$ for each $n \in \mathbb{N}$, we get $\tau(\omega, x)=0$ by the continuity of $y \rightarrow \tau(\omega, y)$. It follows from (7) that

$$
(\omega, x)=[\theta(0, \omega), \varphi(0, \omega) x] \in S
$$

and therefore $x \in S(\omega)$. Thus $S$ is closed.

Lemma 2 Let $(\theta, \varphi)$ be a continuous $R D S$ on $E$ then, the following statements are equivalent

(i) $(\theta, \varphi)$ possesses a continuous random section.

(ii) There exists a measurable mapping $\tau: \Omega \times E \rightarrow \mathbb{R}$ such that $x \mapsto \tau(\omega, x)$ is continuous and

$$
\tau\left(\theta_{t} \omega, \varphi(t, \omega) x\right)=\tau(\omega, x)-t ; \quad(t \in \mathbb{R}, \omega \in \Omega, x \in E) .
$$

Proof. Let $[S, \tau]$ be continuous random section of $(\theta, \varphi)$.

Let $t \in \mathbb{R}, \omega \in \Omega, x \in E$ and put $\bar{\omega}:=\theta(t, \omega) ; \bar{x}:=\varphi(t, \omega) x$. Then the translation equation (1) implies obviously that

$$
\theta(\tau(\omega, x)-t, \bar{\omega})=\theta(\tau(\omega, x)-t, \theta(t, \omega))=\theta(\tau(\omega, x), \omega)
$$

On the other hands, from the cocycle equation (2), we have

$$
\varphi(\tau(\omega, x)-t, \bar{\omega}) \bar{x}=\varphi(\tau(\omega, x)-t, \theta(t, \omega)) \varphi_{t}(\omega) x=\varphi(\tau(\omega, x), \omega) x .
$$

Therefore by (7), (9) and (10) we have

$$
(\theta(\tau(\omega, x)-t, \bar{\omega}), \varphi(\tau(\omega, x)-t, \bar{\omega}) \bar{x}) \in S .
$$


Now, by definitions of $\bar{\omega}, \bar{x}, \tau$ and $S,(11)$ means that (8) holds.

Conversely, let $\tau: \Omega \times E \rightarrow \mathbb{R}$ satisfying (8). Define

$$
S:=\{(\theta(\tau(\omega, x), \omega), \varphi(\tau(\omega, x), \omega) x): \omega \in \Omega, x \in E\}
$$

It remains to prove that $S$ is a random section. To do this, let $L:=\exp (\tau)$ then $(8)$ is equivalent to

$$
L\left(\theta_{t} \omega, \varphi(t, \omega) x\right)=\exp (-t) \cdot L(\omega, x) ; \quad(t \in \mathbb{R}, \omega \in \Omega, x \in E) .
$$

Hence $t \mapsto L\left(\theta_{t} \omega, \varphi(t, \omega) x\right)$ is strictly decreasing and

$$
\lim _{t \rightarrow+\infty} L\left(\theta_{t} \omega, \varphi(t, \omega) x\right)=0 ; \quad \lim _{t \rightarrow-\infty} L\left(\theta_{t} \omega, \varphi(t, \omega) x\right)=+\infty .
$$

In particular, for each $(\omega, x) \in \Omega \times E, \tau(\omega, x)$ is the unique real number such that

$$
L(\theta(\tau(\omega, x), \omega), \varphi(\tau(\omega, x), \omega) x)=1
$$

and therefore $S=\{L=1\}$.

\subsection{Liapunov function}

\section{Definition 2}

(1) A Liapunov function of $(\theta, \varphi)$ is a measurable function $L: \Omega \times E \rightarrow \mathbb{R}$ such that, for all $t \geq 0, \omega \in$ $\Omega, x \in E$ :

$$
L\left(\theta_{t} \omega, \varphi(t, \omega) x\right) \leq L(\omega, x)
$$

(i.e. $L$ is decreasing over all orbits of $(\theta, \varphi)$ ).

(2) A Liapunov function $L$ is said to be strict if

$$
L\left(\theta_{t} \omega, \varphi(t, \omega) x\right) \neq L(\omega, x)
$$

for all $\omega \in \Omega, x \in E, t \neq 0$. (i.e. $L$ is strictly decreasing over all orbits of $(\theta, \varphi)$ ).

(3) A Liapunov function is said to be continuous if, for each $\omega \in \Omega$, the function $x \mapsto L(\omega, x)$ is continuous.

Theorem 1 Let $(\theta, \varphi)$ be a continuous $R D S$ on $E$ then, the following statements are equivalent

(i) $(\theta, \varphi)$ possesses a continuous random section.

(iii) $(\theta, \varphi)$ admits a Liapunov function which is continuous and strict.

Proof: Let $[S, \tau]$ be continuous random section of $(\theta, \varphi)$. It follows from Lemma 2 that $L:=\exp (\tau)$ is a strict and continuous Liapunov function of $(\theta, \varphi)$ since (13) implies trivially (14) and (15).

Conversely, let $L: \Omega \times E \rightarrow] 0, \infty[$ be a strict and continuous Liapunov function of $\varphi$. Define, for $(\omega, x) \in \Omega \times E$,

$$
\tilde{L}(\omega, x):=\int_{0}^{\infty} \exp (-r) L\left(\theta_{r} \omega, \varphi(r, \omega) x\right) d r
$$

While $L$ is measurable then so is $\tilde{L}$. Moreover by the continuity of $x \mapsto \varphi(r, \omega) x$ and $x \mapsto L(\omega, x)$, we deduce the continuity of $x \mapsto \tilde{L}(\omega, x)$ using a classical continuity result.

On the other hand, by the translation and the cocycle equations (1) and (2), it follows that

$$
\tilde{L}\left(\theta_{t} \omega, \varphi(t, \omega) x\right)=\exp (t) \int_{t}^{\infty} \exp (-r) L\left(\theta_{r} \omega, \varphi(r, \omega) x\right) d r
$$


for $t \in \mathbb{R}, \omega \in \Omega$ and $x \in E$. Or equivalently

$$
\exp (-t) \tilde{L}\left(\theta_{t} \omega, \varphi(t, \omega) x\right)=\tilde{L}(\omega, x)-\int_{0}^{t} \exp (-r) L\left(\theta_{r} \omega, \varphi(r, \omega) x\right) d r
$$

Let

$$
G(t, \omega, x):=\exp (-t) \tilde{L}\left(\theta_{t} \omega, \varphi(t, \omega) x\right)
$$

From (16) we obtain first that $t \mapsto G(t, \omega, x)$ is decreasing, second that $(r, x) \rightarrow G(r, \omega, x)$ is continuous and third

$$
\lim _{t \rightarrow+\infty} G(t, \omega, x)=0 ; \quad \lim _{t \rightarrow-\infty} G(t, \omega, x)=+\infty .
$$

Therefore, for each $(\omega, x) \in \Omega \times E$, there exists by (17), a unique $\tau(\omega, x) \in \mathbb{R}$ such that

$$
G(\tau(\omega, x), \omega, x)=1
$$

Hence $\tau: \Omega \times E \rightarrow \mathbb{R}$ is well defined and by putting (as in the proof of Lemma 2)

$$
S:=\{(\theta(\tau(\omega, x), \omega), \varphi(\tau(\omega, x), \omega) x):(\omega, x) \in \Omega \times E\}
$$

we define a random section $[S, \tau]$ of the $\operatorname{RDS} \varphi$.

It remains to prove that $x \mapsto \tau(\omega, x)$ is continuous. Let $\omega \in \Omega, x \in E$ and $\left(x_{n}\right)$ a net of $E$ which tends to $x$. There are only two possibilities:

(1) The sequence $\left(\tau\left(\omega, x_{n}\right)\right)$ is bounded. Let $\left(y_{n}\right)$ and $\left(z_{n}\right)$ be two subsequences of $\left(x_{n}\right)$ such that $\left(\tau\left(\omega, y_{n}\right)\right)$ and $\left(\tau\left(\omega, z_{n}\right)\right)$ are convergent to $s$ and $t$ respectively. Applying (18) to $(\omega, x),\left(\omega, x_{n}\right)$ and $\left(\omega, z_{n}\right)$ we get

$$
G(\tau(\omega, x), \omega, x)=G\left(\tau\left(\omega, y_{n}\right), \omega, y_{n}\right)=G\left(\tau\left(\omega, y_{n}\right), \omega, y_{n}\right)
$$

Letting $n \rightarrow \infty$ in (19), we conclude that $s=t=\tau(\omega, x)$ by continuity of $(r, x) \mapsto G(r, \omega, x)$ and by injectivity of $r \mapsto G(r, \omega, x)$.

(2) The sequence $\left(\tau\left(\omega, x_{n}\right)\right)$ is unbounded. There exists in this case a subsequence $\left(z_{n}\right)$ of $\left(x_{n}\right)$ such that $\left(\tau\left(\omega, z_{n}\right)\right) \uparrow+\infty$ or such that $\left(\tau\left(\omega, z_{n}\right)\right) \downarrow-\infty$. If $\left(\tau\left(\omega, z_{n}\right)\right) \uparrow+\infty$, define

$$
f_{n}(y):=G\left(\tau\left(\omega, z_{n}\right), \omega, y\right) ; \quad(y \in E)
$$

then $\left(f_{n}\right)$ is a decreasing sequence of functions which converges simply to 0 in view of $(17)$. Hence $\left(f_{n}\right)$ converges locally uniformly to 0 . While $\left(z_{n}\right)$ belong to compact neighborhood of $x$ and $\lim f_{n}(x)=0$ we must have $\lim G\left(\tau\left(\omega, z_{n}\right), \omega, z_{n}\right)=0$. But from (19) we derive $\lim G\left(\tau\left(\omega, z_{n}\right), \omega, z_{n}\right)=1$ and we have arrived at a contradiction.

If $\left(\tau\left(\omega, z_{n}\right)\right) \downarrow-\infty$, we consider

$$
g_{n}(y):=\frac{1}{G\left(\tau\left(\omega, z_{n}\right), \omega, y\right)} ; \quad(y \in E)
$$

instead of $f_{n}$ and, by the same arguments, we obtain again a contradiction.

Remark 3 If we take $\Omega=\{\varpi\}$ (i.e. there is no noise), then $\varphi$ is a continuous (deterministic) DS on $E$. In this case, the preceding characterization is known (cf. [5], Theorem 19). Moreover, the preceding proof is partially adapted from that of [5]. 


\subsection{Real cocycle of RDS}

Definition 3 Let $(\theta, \varphi)$ be a continuous RDS on $E$. An associated real cocycle is a measurable mapping $C: \mathbb{R} \times \Omega \times E \rightarrow] 0, \infty[$ such that

(1) $(t, x) \mapsto C(t, \omega, x)$ is continuous.

(2) $C$ satisfies the equation:

$$
C(0, \omega, x)=1, \quad C(s+t, \omega, x)=C\left(s, \theta_{t} \omega, \varphi(t, \omega) x\right) \cdot C(t, \omega, x) .
$$

In other words $C$ is a cocycle over the skew product DS $\Phi$ and with state space ]0, $\infty[$.

The deterministic counter-part of the next result is proved in [6] (cf. Lemma 3.2). The proof below is adapted from $[6]$.

Theorem 2 Let $(\theta, \varphi)$ be a continuous RDS on $E$ then, the following statements are equivalent

(i) $(\theta, \varphi)$ possesses a continuous random section.

(iv) Each real cocycle $C$ of $(\theta, \varphi)$, is of the form

$$
C(t, \omega, x)=\frac{u(\omega, x)}{u\left(\theta_{t} \omega, \varphi(t, \omega) x\right)}
$$

for some measurable $u: \Omega \times E \rightarrow] 0, \infty[$ and such that $x \mapsto u(\omega, x)$ is continuous.

Proof. According to Lemma 2, it is enough to prove the equivalence between (ii) and (iv).

Let $\tau: \Omega \times E \rightarrow] 0, \infty$ [ satisfying (ii) and let $C$ be a real cocycle of $(\theta, \varphi)$. Define

$$
u(\omega, x):=C(\tau(\omega, x), \omega, x) ; \quad(\omega \in \Omega, x \in E) .
$$

Clearly $u$ is measurable and $x \mapsto u(\omega, x)$ is continuous. Further, from (2) and (8) we derive (21) since

$$
\begin{aligned}
u\left(\theta_{t} \omega, \varphi(t, \omega) x\right) & =C\left(\tau\left(\theta_{t} \omega, \varphi(t, \omega) x\right), \theta_{t} \omega, \varphi(t, \omega) x\right) \\
& =C\left(\tau(\omega, x)-t, \theta_{t} \omega, \varphi(t, \omega) x\right) \\
& =\frac{u(\omega, x)}{C(t, \omega, x)} .
\end{aligned}
$$

Conversely, notice that $(t, \omega, x) \mapsto C(t, \omega, x):=\exp (t)$ is real cocycle of $(\theta, \varphi)$. Then there is a measurable $u$ such that $x \mapsto u(\omega, x)$ is continuous and

$$
\exp (t)=\frac{u(\omega, x)}{u\left(\theta_{t} \omega, \varphi(t, \omega) x\right)}
$$

We conclude exactly as in the proof of Lemma 2 .

\subsection{Gradient-like RDS}

Definition 4 A continuous $\operatorname{RDS}(\theta, \varphi)$ is said to be gradient-like, il there exists a random set $S \subset \Omega \times E$ and a homemorphism $h: \Omega \times E \rightarrow \mathbb{R} \times S$ such that

(1) For each $(\omega, x) \in \Omega \times E$, there exists $\bar{t} \in \mathbb{R},(\bar{\omega}, \bar{x}) \in S$ such that

$$
(\theta(\bar{t}, \bar{\omega}), \varphi(\bar{t}, \bar{\omega}) \bar{x})=(\omega, x) .
$$

(2) For all $t \in \mathbb{R},(\bar{\omega}, \bar{x}) \in S$

$$
h\left(\theta_{t} \bar{\omega}, \varphi(t, \bar{\omega}) \bar{x}\right)=(t,(\bar{\omega}, \bar{x})) .
$$


In this case, we say that $(\theta, \varphi)$ is generated by $S$.

Theorem 3 Let $(\theta, \varphi)$ be a continuous RDS on $E$ then, the following statements are equivalent

(i) $(\theta, \varphi)$ possesses a continuous random section.

(v) $(\theta, \varphi)$ is a gradient-like $R D S$.

Proof. Let $[S, \tau]$ be a continuous random section. Define $h: \Omega \times E \rightarrow \mathbb{R} \times S$ by

$$
h(\omega, x):=(-\tau(\omega, x),(\theta(\tau(\omega, x), \omega), \varphi(\tau(\omega, x), \omega) x) .
$$

It follows from (1), (2) and (8) that

$$
h\left(\theta_{t} \omega, \varphi(t, \omega) x\right):=(t-\tau(\omega, x),(\theta(\tau(\omega, x), \omega), \varphi(\tau(\omega, x), \omega) x) .
$$

If $(\omega, x) \in S$ then $\tau(\omega, x)=0$ and therefore (23) appears as a particular case of (25).

Now, $h$ is one-to-one by definition of the section $[S, \tau]$. Moreover, $h$ is measurable (as composition of measurable mappings). Further, for every $\omega \in \Omega, x \mapsto h(\omega, x)$ is continuous by the continuity of $x \mapsto \tau(\omega, x)$ and of $x \mapsto \varphi(t, \omega) x$.

On the other hand, let $\bar{t} \in \mathbb{R}$ and $(\bar{\omega}, \bar{x}) \in S$. By resolving the system $h(\omega, x)=(\bar{t},(\bar{\omega}, \bar{x}))$, it can be verified that

$$
h^{-1}(\bar{t},(\bar{\omega}, \bar{x}))=(\theta(\bar{t}, \bar{\omega}), \varphi(\bar{t}, \bar{\omega}) \bar{x})
$$

and therefore $(22)$ is satisfied. We conclude that $\varphi$ is gradient-like.

Conversely, suppose that $\varphi$ is gradient-like. Then the random set $S$ in its definition, is a continuous random section of $\varphi$. Indeed, let $(\omega, x) \in \Omega \times E$ then there exists $\bar{t} \in \mathbb{R}$ and $(\bar{\omega}, \bar{x}) \in S$ (necessary unique) such that (22) holds. We set $\tau(\omega, x):=-\bar{t}$. Applying $h$ on the equality (22) and using (3), (23) and (26), we obtain the formula (24) by solving the system

$$
(\omega, x)=(\theta(\bar{t}, \bar{\omega}), \varphi(\bar{t}, \bar{\omega}) \bar{x})
$$

In particular, $\tau$ is measurable and $x \mapsto \tau(\omega, x)$ is continuous by the measurability of $h$ and the continuity of $x \mapsto h(\omega, x)$.

Remark 4 If we take $\Omega=\{\varpi\}$ (i.e. there is no noise), then $\varphi$ is a continuous (deterministic) DS on $E$. In this case, the preceding characterization is known (cf. [2] Theorem 2.4). Moreover, the preceding proof is adapted from that of the deterministic case.

Example 3 Let $\theta$ be a DS over a measurable space $(\Omega, \mathcal{F})$.

Consider the Lorenz system in $\mathbb{R}^{3} \backslash\{(0,0,0)\}$ perturbed by real noises:

$$
(R D E) \quad\left\{\begin{aligned}
\dot{u} & =\sigma\left(\theta_{t} \omega\right)(v-u) \\
\dot{v} & =\rho\left(\theta_{t} \omega\right) u-v-u w \\
\dot{w} & =u v-\beta\left(\theta_{t} \omega\right) w
\end{aligned}\right.
$$

such that

(1) $\sigma(\omega), \rho(\omega), \beta(\omega)>0$.

(2) $\rho(\omega)<\sigma(\omega) \leq 1$.

Then the (RDE) generates an $\operatorname{RDS} \varphi$ (cf. $[1,9]$ for details).

Let

$$
L(\omega, x, y, z)=x^{2}+y^{2}+z^{2} .
$$


By derivation of $L$ w.r.t $t$ along orbits of $\varphi$, we obtain

$$
\dot{L}=-(\sigma-\rho) u^{2}-(2-\rho-\sigma) v^{2}-2 \beta w^{2}<0 .
$$

Therefore, for all $x, y, z \in \mathbb{R}^{3} \backslash\{(0,0,0)\}$ and $t>0$ :

$$
L\left(\theta_{t} \omega, \varphi_{t}(\omega)(x, y, z)\right)<L(\omega, x, y, z) .
$$

We conclude that $(\theta, \varphi)$ is gradient-like.

\subsection{Parallelizable RDS}

Definition 5 A continuous RDS $(\theta, \varphi)$ with state space $E$ is said to be parallelizable if it is random conjugate to a parallel RDS $(\theta, \pi)$ with state space $\mathbb{R} \times Y$ for some closed subset $Y$ of $E$.

Theorem 4 Let $(\theta, \varphi)$ be a continuous RDS on $E$ then, the following statements are equivalent

(1) $(\theta, \varphi)$ is gradient-like generated by a band as random section.

(2) $(\theta, \varphi)$ is parallelizable.

Proof. Let $Y$ be closed subset of $E$ and $\tau: \Omega \times E \rightarrow \mathbb{R}$ such that $[\tau, \Omega \times Y]$ is a continuous random section of $(\theta, \varphi)$ (cf. theorem 2). Let $h: \Omega \times E \rightarrow \mathbb{R} \times \Omega \times Y$ be given by (24). We define $f: \Omega \times E \rightarrow \mathbb{R} \times Y$ as the projection of $h$ on $\mathbb{R} \times Y$, i.e.

$$
f(\omega, x):=(-\tau(\omega, x), \varphi(\tau(\omega, x), \omega) x) ; \quad(\omega \in \Omega, x \in E) .
$$

Then

(a) For each $\omega \in \Omega$, the mapping $x \mapsto f(\omega, x)$ is an homeomorphism from $E$ onto $\mathbb{R} \times Y$. Indeed $f$ is surjective since $h$ is surjective. On the other hand, let $x_{1}, x_{2} \in E$ such that $f_{\omega}\left(x_{1}\right)=f_{\omega}\left(x_{2}\right)$. It follows by (27) that $\tau\left(\omega, x_{1}\right)=\tau\left(\omega, x_{1}\right)$ and therefore $h\left(\omega, x_{1}\right)=h\left(\omega, x_{2}\right)$ by definition of $h$ (relation 24). Thus $x_{1}=x_{2}$ since $h$ is injective.

(b) For each $x \in E$, the mapping $\omega \mapsto f(\omega, x)$ is clearly measurable.

On the other hand, let $y \in Y, r \in \mathbb{R}$. It follows by (3) and (27) that

$$
f_{\omega}^{-1}(r, y)=\varphi\left(-r, \theta_{r} \omega\right) y
$$

Hence $\omega \mapsto f_{\omega}^{-1}(r, y)$ is measurable.

(c) As in the proof of theorem 2, we derive

$$
f\left(\theta_{t} \omega, \varphi(t, \omega) x\right)=(t+\tau(\omega, x), \varphi(\tau(\omega, x), \omega) x)
$$

for all $\omega \in \Omega, x \in E, t \in \mathbb{R}$. However, by definition of $f$ (relation (27)) and $\pi$ (relation (4)), we get

$$
(t+\tau(\omega, x), \varphi(\tau(\omega, x), \omega) x)=\pi(t, \omega) f(\omega, x) .
$$

Combining (28) and (29) we obtain (5) and conclude that $\varphi$ et $\pi$ are RDS-isomorph.

Conversely, let $\pi$ be a parallel RDS generated by a closed subset $Y$ of $E$ and let $f: \Omega \times E \rightarrow \mathbb{R} \times Y$ satisfying (a), (b) and (c) of the definition of random isomorphism. If we write $f(\omega, x):=(r(\omega, x), z(\omega, x))$ then we define $\tau: \Omega \times E \rightarrow \mathbb{R}$ by $\tau(\omega, x):=-r(\omega, x)$. Then like $f, \tau$ is measurable and $x \mapsto f(\omega, x)$ is continuous by (i).

Further, by (c), we get

$$
f\left(\theta_{t} \omega, \varphi(t, \omega) x\right)=(t+r(\omega, x), z(\omega, x))=(t-\tau(\omega, x), z(\omega, x))
$$


which implies again (8), that is $\tau\left(\theta_{t} \omega, \varphi(t, \omega) x\right)=\tau(\omega, x)-t$.

Arguing as in the proof of Lemma 2 (which use essentially (8)), we prove that $[\tau, \Omega \times Y]$ is a continuous random section of $(\theta, \varphi)$. We conclude that $(\theta, \varphi)$ is parallelizable.

Remark 5 It follows from theorem 4 that, a parallelizable RDS is a gradient-like RDS such that an associated random section is a band. However, for the deterministic case, there is no difference between gradient-like and parallelizable dynamical systems.

\subsection{Backward cocycle}

Let $\theta$ be a DS on $(\Omega, \mathcal{F})$ and let $(E, \mathcal{E})$ be a LCCB space.

A cocycle $\varphi: \mathbb{R} \times \Omega \times E \rightarrow E$ over $\theta$, is in fact called a forward cocycle over $\theta$.

A backward cocycle on $E$ over the DS $\theta$, is a map $\psi: \mathbb{R} \times \Omega \times E \rightarrow E,(t, \omega, x) \mapsto \psi(t, \omega) x$ such that

(1) $\psi$ is $(\mathcal{R} \otimes \mathcal{F} \otimes \mathcal{E}, \mathcal{E})$-measurable.

(2) $(t, x) \rightarrow \psi(t, \omega) x$ is continuous for each $\omega \in \Omega$.

(3) $\psi$ satisfies the backward cocycle equation, i.e.: For $s, t \in \mathbb{R}, \omega \in \Omega, x \in E$ :

$$
\psi(0, \omega)=I_{E}, \quad \psi(s+t, \omega)=\psi(s, \omega) \circ \psi\left(t, \theta_{s} \omega\right) x .
$$

The proof of the following equivalence is an immediate consequence of (3) and (30).

Lemma 3 The following statements are equivalent.

(1) $\varphi$ is a forward cocycle over $\theta$

(2) $\psi:=\varphi^{-1}$ is a backward cocycle over $\theta$.

All notions introduced above (gradient-like, random section, Liapunov function, ...) can be easily defined for backward RDS.

As consequence of theorems 1 and 3, we deduce the following result.

Corollary With the same notations as in Lemma 3, the following statements are equivalent

(v) $(\theta, \varphi)$ is a gradient-like RDS.

(vi) $(\theta, \eta)$ is a gradient-like $R D S$.

Proof. It suffices to remark that $L$ is a Liapunov function for $(\theta, \varphi)$ if and only if $1 / L$ is a Liapunov function for $(\theta, \eta)$.

\section{Remarks 5}

(1) Notice that $\eta(t, \omega):=\varphi(-t, \omega)$ and $\chi(t, \omega):=\varphi(-t, \omega)^{-1}$ are both backward cocycles over $\theta^{-1}$. Hence $(\mathrm{v})$ is therefore equivalent to $\left(\theta^{-1}, \psi\right)$ is a gradient-like $R D S$ or to $\left(\theta^{-1}, \chi\right)$ is a gradient-like $R D S$.

(2) In the corollary, we may replace gradient-like by parallelizable.

(3) If there is no noise, this result is known (cf. [5], Corollary 16 and Theorem 19).

Final Remark We close this paper by the following remark concerning general RDS: Under some additional conditions on $(\Omega, \mathcal{F})$, any $\operatorname{RDS}(\theta, \varphi)$ with state space $E$, decomposes $E$ into a random chain recurrent part and a random gradient-like part (cf. [9] Theorem 8).

Hence, our results may be applied for the gradient-like part for general RDS.

For example, the Lorenz system (cf. Example 3 above) could be defined in the whole space $\mathbb{R}^{3}$. In this case, $\{(0,0,0)\}$ is the associated random chain recurrent part and $\mathbb{R}^{3} \backslash\{(0,0,0)\}$ is the random gradient-like part. Many other examples could be obtained in this way. 


\section{REFERENCES}

[1] Arnold L.: Random Dynamical Systems, Springer-Verlag, Berlin (1998).

[2] Bhatia N.P., Szego G.P.: Stability Theory of Dynamical Systems, Grundl. Math. Wiss. 161, Springer-Verlag, (1970).

[3] Chen X., Duan J., Fu X.: A sufficient condition for bifurcation in random dynamical systems, Proc. Amer. Math. Soc. 138, No 3, 965-973 (2010).

[4] Cornfeld I.P., Fomin S.V., Sinai Y.G.: Ergodic Theory, Grundl. math. Wissensch. 245, Springer-Verlag, Berlin Heidelberg New-York (1982).

[5] Hmissi. M.: Semi-groupes déterministes, Lecture notes in Math. 1393, Springer-Verlag, 135-144 (1989).

[6] Hmissi, M.: Sur les solutions globales de l'équation des cocycles, Aequ. Math. 45, 195-206 (1993).

[7] Hmissi. M.: On the exit equation for semidynamical systems, Grazer Math. Berichte 339, 221-232 (1999).

[8] Kloeden, P.E.: A Lyapunov function for pullback attractors of nonautonomous differential equations, Nonlinear Differential Equations, Electron. J. Diff. Eqns., Conf. 05, 91-102 (2000).

[9] Liu Z.: The random case of Conley's theorem II: The complete Liapunov function, Nonlinearity 20 (2007).

[10] Whitney H.: Regular families of curves, Ann. of Math. 34, 244-270 (1933). 